This item is the archived peer-reviewed author-version of:

Pulmonary nodule detection in chest ct using a deep learning-based reconstruction algorithm

\title{
Reference:
}

Franck Caro, Snoeckx Annemie, Spinhoven Maarten, El Addouli Haroun, Nicolay S., Van Hoyweghen A., Deak P., Zanca F..- Pulmonary nodule detection in chest ct using a deep learning-based reconstruction algorithm

Radiation protection dosimetry - ISSN 0144-8420 - (2021)17 p.

Full text (Publisher's DOI): https://doi.org/10.1093/RPD/NCAB025

To cite this reference: https://hdl.handle.net/10067/1802860151162165141 


\section{Pulmonary nodule detection in chest CT using a deep learning-based reconstruction algorithm}

C. Franck ${ }^{1,2 *}$, A. Snoeckx ${ }^{1,2}$, M. Spinhoven ${ }^{1,2}$, H. El Addouli ${ }^{1,2}$, S. Nicolay ${ }^{1,2}$, A. Van Hoyweghen ${ }^{1,2}$, P. Deak ${ }^{3}$ and F. Zanca ${ }^{4}$

*corresponding author: caro.franck@uza.be

1 Department of Radiology

University Hospital Antwerp

Drie Eikenstraat 655

2650 Edegem, Belgium

2 mVISION, Faculty of Medicine and Health Sciences

University of Antwerp

Drie Eikenstraat 655

2650 Edegem, Belgium

3 GE Healthcare

Glattbrugg, Switzerland

$4 \quad$ Palindromo Consulting

Leuven, Belgium

\section{Short title}

Nodule detection using DL reconstruction 


\begin{abstract}
This study's aim was to assess whether deep learning image reconstruction (DLIR) techniques are noninferior to ASIR-V for the clinical task of pulmonary nodule detection in chest CT. Up to 6 (range 3-6, mean 4.2) artificial lung nodules (diameter: $3,5,8 \mathrm{~mm}$; density: $-800,-630,+100 \mathrm{HU}$ ) were inserted at different locations in the Kyoto Kagaku Lungman phantom. In total, 16 configurations ( 10 abnormal, 6 normal) were scanned at $7.6 \mathrm{mGy}, 3 \mathrm{mGy}, 1.6 \mathrm{mGy}$ and $0.38 \mathrm{mGy}$ CTDIvol (respectively 0\%, 60\%, 80\% and 95\% dose reduction). Images were reconstructed using 50\% ASIR-V and a deep learning-based algortihm with low (DL-L), medium (DL-M) and high (DL-H) strength. Four chest radiologists evaluated 256 series by locating and scoring nodules on a five-point scale. No statistically significant difference was found among the reconstruction algorithms ( $p=0.987$, average across readers AUC: 0.555, 0.561, 0.557, 0.558 for ASIR-V, DL-L, DL-M, DL-H).
\end{abstract}

\title{
Attributes
}

Computed Tomography, Cancer, Diagnostic Radiology, Medical, Phantoms, Readers 


\section{INTRODUCTION}

A pulmonary nodule is a rounded opacity in the lung, well or poorly defined, measuring up to $3 \mathrm{~cm}$ in diameter ${ }^{1}$. The differential diagnosis of pulmonary nodules is broad, including benign and malignant etiologies. Identification of these nodules that might present early lung cancers is important since stage at diagnosis is crucial for prognosis ${ }^{2}$. Size, growth and morphology are key factors in assessing the malignant potential of a nodule ${ }^{3,4}$. Subsequent the widespread use of MDCT, small pulmonary nodules are more frequently detected. Furthermore, there is a growing interest in lung cancer screening (LCS) since large randomized controlled trials have shown that lung cancer screening with low-dose CT (LDCT) in high-risk participants can reduce lung cancer-specific mortality. The National Lung Screening Trial (NLST) showed a $20 \%$ lung cancer specific mortality reduction in participants who were screened with LDCT, compared to the control group which was screened with chest radiography ${ }^{5}$. Final data of the randomized Dutch-Belgian NELSON (Nederlands-Leuvens Longkanker Screenings Onderzoek) LCS trial, recently showed a lung cancer mortality reduction of $24 \%$ in men and $33 \%$ in women at 10 -year followup ${ }^{6}$. A $39 \%$ reduction of lung cancer mortality after 10 years of screening was found in the Italian MILD (Multicentric Italian Lung Detection) Trial ${ }^{7}$. The German LUSI (Lung Cancer Screening Intervention) Trial found a non-significant lung cancer-specific mortality reduction of $6 \%$ in men, but a significant reduction in lung cancer-specific mortality of $69 \%$ in women after an average of 8.8 years of follow-up ${ }^{8}$. Recently, the European Respiratory Society and European Society of Radiology published a statement paper on LCS, calling to convince policymakers across the European Union to start implementation of high-quality LCS programs $^{9,10}$.

In an effective screening program for an asymptomatic population, the potential benefits must outweigh harmful effects. One of the potential harms of LCS is the use of radiation ${ }^{11}$. LDCT should allow identification of pulmonary nodules using as low a radiation dose as can reasonably be achieved (ALARA). This is possible using lower tube currents $(\mathrm{mA})$ and lower tube voltages $(\mathrm{kV})$. The NLST decreased radiation dose through lowering tube current ${ }^{5}$.

Unfortunately, image noise is proportionally increased by decreasing the radiation dose. Iterative reconstruction (IR) techniques have been developed to reduce noise associated with dose reduction and subsequently to improve image quality. When compared to the filtered back projection images however, the look and feel of these iterative reconstruction images is very different ${ }^{12,13}$. Radiologists experience these IR-images as more 'plastic-looking' and might feel less confident with their diagnosis ${ }^{14}$. This might impact the utilization of LDCT protocols. 
Recently, deep learning image reconstruction (DLIR) methods have been proposed ${ }^{15}$. These new reconstruction techniques open a new era for CT-image reconstruction by addressing challenges of FBP and IR. DLIR features a deep neural network (DNN), which was trained with high quality FBP datasets to learn how to differentiate noise from signals, and to intelligently suppress the noise without impacting anatomical and pathological structures. The purpose of this study was to assess whether detection of pulmonary nodules on low-dose CT with DLIR is non-inferior to detection on low-dose CT with IR techniques, at comparable or even lower dose levels than IR reconstructions. 


\section{MATERIALS AND METHODS}

\section{Phantom selection and preparation}

The Multipurpose Chest Phantom N1 "LUNGMAN" (Kyoto Kagaku) was used for our experiments. The phantom (Figure 1) is made of polyurethane and is composed of a male chest torso, in which synthetic bones (epoxy resins) are embedded, plus four internal parts: mediastinum (heart, trachea), pulmonary vessels and bronchial branches (right and left) and abdomen (diaphragm). Two $30 \mathrm{~mm}$ fat slabs were added to the phantom, to represent a torso of an $82 \mathrm{~kg}$ and $168 \mathrm{~cm}$ male (body-mass index of 29). A senior chest radiologist, who was not involved in the observer performance experiment, laced up the phantom with simulated lesions having 3 varieties of Hounsfield Unit (HU) number (approx. $-800,-630,+100$ ) and available in 3 sizes for each type (diameters 3, 5, $8 \mathrm{~mm}$ ), shown in Figure 1. These HU cover the spectrum of both solid and and ground glass lung nodules: the -800 and $-630 \mathrm{HU}$ densities represent nonsolid nodules (type pure ground glass) in clinical practice. The nodule with density of $+100 \mathrm{HU}$ represents a solid nodule. Out of these 9 sphere-shaped lesions, 16 different configurations were identified to lace up the phantom: ten consisted of up to 6 (range 3-6) nodules placed in the pulmonary vasculature in a random order, and an additional 6 were without nodules (Table 1).

\section{Image acquisition and reconstructions}

The laced-up phantom with the different configurations was imaged using a 256-slice Revolution CT (GE Healthcare) and our clinical chest protocol (120 kVp; $\left.7.6 \mathrm{mGy} C T D I_{v o l}\right)$, with the midline of the phantom positioned at isocenter. Three (3) additional dose levels were acquired: $3 \mathrm{mGy}, 1.6 \mathrm{mGy}$ and $0.4 \mathrm{mGy}$ CTDI $_{\text {vol, }}$ resulting in $60 \%, 80 \%$ and $95 \%$ dose reduction respectively, to possibly represent low-dose chest CT. Other scanning parameters included: rotation time, $350 \mathrm{~ms}$; collimation, $40 \mathrm{~mm}$ and pitch, 0.98 .

Consecutive slices of $1.25 \mathrm{~mm}$ were reconstructed with a STANDARD kernel using iterative reconstruction (ASIR-V) at $50 \%$ blending, which is the clinical standard in our hospital. In addition, images were reconstructed using a DL-based algorithm (TrueFidelity) with low (L), medium (M) and high (H) strength ${ }^{15}$. $L, M, H$, are three selectable reconstruction modes that correspond on how the image noise is modeled. While of radiologist preference, for high contrast tissue (e.g. enhanced scans with increased iodine uptake on relatively large areas) a more aggressive noise reduction provided by DL-H has been observed to be of preference, for density difference, (e.g. low contrast detectability) medium to low are generally used in clinical practice. This DLIR algorithm, build upon knowledge of the CT system design, is embedded within a Deep Neural Network (DNN). The DNN is trained by feeding a low-dose sinogram through network and 
comparing the output image to a ground truth image - a high dose version of the same data; millions of parameters representing the DNN are fine-tuned through embedded backpropagation based on those differences. The comparison occurs over multiple parameters such as image noise, low contrast resolution, low contrast detectability, noise texture, etc. This process is repeated until the DLIR is able to generate ground truth equivalent high-quality $\mathrm{CT}$ images, from an input sinogram that is acquired with low radiation dose. TrueFidelity was first introduced in our department through this study so that no preferred strength existed. In total 256 CT series were generated (16 nodule configurations, 4 dose levels, 4 reconstruction algorithms). The series were randomized and blinded for dose and reconstruction parameters.

\section{Observer performance experiment}

The observer performance experiment used the freeresponse paradigm ${ }^{16}$. Four radiologists ( 3 to 12 years of experience in chest imaging), were asked to detect and locate the perceived nodules. The observers were told that each image could contain zero or more nodules. A five points rating scale was used: $1=$ highly unlikely to be a nodule, $2=$ unlikely to be a nodule, $3=$ somewhat confident it is a nodule, 4=moderately confident it is a nodule, and $5=$ very confident it is a nodule. A clinically reportable lesion would be assigned at a score $\geq 3$. By clicking on the lesion, the location of the cursor $(x, y, z)$ was stored and exported at the end of each reading session. Next, the location was compared with the ground truth coordinates of the nodules in that specific Lungman configuration, as previously defined by an experienced radiologist who did not participate in the reading sessions.

Images were presented on a clinical PACS environment using a 30 inch, 4 MP high-contrast color monitor (Barco MDCC-4430) at optimal lighting conditions, according to the AAPM TG18 report ${ }^{17}$. A training session ( 25 images) preceded the evaluation in order to familiarize the radiologists with the user interface and the processing algorithms. The reading sessions were performed according to each user's availability, but each session would not last more than $2 \mathrm{~h}$ in order to reduce fatigue effects on the results. Within each session, images processed with each of the four algorithms were presented randomly.

\section{Statistical analysis}


The primary analysis of the free-response data obtained from this multiple-reader multiple-case (MRMC) study was JAFROC, using version JAFROC1_V1 available from http://www.devchakraborty.com. Prior to analysis, each mark had to be classified as a lesion localization (LL) or non-lesion localization (NL), by using the ground truth file generated as described earlier. Specifically, if the observer clicked anywhere within a range of 1.5 times the nodule radius, measured from the center of the nodule, the case was considered a LL, outside this acceptance radius, each case was considered a NL. The size of the acceptance radius was therefore nodule dependent.

Data analysis consists of estimating the JAFROC figure of merit (FoM) and a method for testing the statistical significance of differences in figures of merit between image processing algorithms. The JAFROC FoM is defined as the probability that a lesion is rated higher than all non-lesions on normal images and the significance testing is performed using the ANOVA procedure. The presented analysis treats both readers and cases as random samples, such that results apply to the reader and case populations. Figures of merit, F-statistics, degrees of freedom, $p$-values, and $95 \%$ confidence intervals are reported ${ }^{16}$. 


\section{RESULTS}

The overall results of JAFROC analyses are summarized in Table 2 which lists the F-statistics, the numbers of degrees of freedom (numerator/denominator) and the p-value. A p-value less than 0.05 means that the differences in Figures of Merit (FoMs) between at least two image reconstruction algorithms pairings are statistically significant. JAFROC yielded $F=0.05, p=0.987$. No statistically significant difference was found among the four reconstruction algorithms.

Table 3 reports the FoMs for JAFROC for each algorithm; listed are values for each reader and the average of all the readers (last row). The DL algorithms yielded a slightly higher figure of merit than the ASIR-V $50 \%$ but the difference was not significant.

Table 4 reports the differences in figures of merit between all modality pairings as well as the $95 \%$ confidence intervals for these differences. If a confidence interval does not include zero, the corresponding difference is statistically significant, which was never the case for our data. Corrections for multiple comparisons are automatically considered in the ANOVA analysis. 


\section{DISCUSSION}

With the increased use of CT and advent of large LCS trials, low-dose imaging of the lungs and implementation of LDCT protocols in clinical practice has gained interest. Dose reduction is especially important in screening, since it is applied to a large number of participants, who are also asymptomatic. Regarding methods to reduce dose, the performance of IR methods in terms of noise and dose reduction has been extensively researched ${ }^{14,18,19}$. Although dose savings ranging from $30 \%$ to $80 \%$ are reported in literature ${ }^{14,19}$, IR methods are often described as having an overly smooth or synthetic noise impression. The low frequency noise texture typically associated with IR techniques has shown to be a clinical limitation for some imaging tasks ${ }^{20}$. Indeed, it is known in literature that, despite a significant noise reduction compared to FBP methods, a frequency shift in the noise power spectrum (NPS) is present for iterative reconstruction algorithms ${ }^{21-23}$. Recently, deep learning image reconstruction (DLIR) methods have been proposed ${ }^{15}$. These new reconstruction techniques allow reduction of radiation dose, while preserving the image quality of the FBP technique. Several studies reported similar noise texture in DL and FBP reconstructions, based on the comparison of noise power spectra in physical-technical phantoms ${ }^{24-26}$. Little or no research on the impact of low-dose DL reconstructions on clinical tasks can be found in literature ${ }^{27,28}$. To our knowledge, our work represents the first pulmonary nodule detection study on DL reconstructed images (TrueFidelity) of low-dose chest CT.

The aim of this study was to assess whether detection of pulmonary nodules of different densities, on low-dose CT with DLIR is non-inferior to detection on low-dose CT with IR techniques, at comparable or even lower dose levels than IR reconstructions.

Our results show an excellent interobserver agreement in nodule detection in terms of JAFROC figure of merit between all four radiologists (Table 3). DL algorithms yielded a slightly higher figure of merit than ASIR-V 50\%, yet differences were not significant. A possible explanation could be the considered clinical task of searching for several artificial nodules which are not fully representative of the typical clinical scenario. The absolute difference in HU between the lesions and the background (air: -1000 HU) was 200, 370 and $1100 \mathrm{HU}$, for the low, medium and high density nodules respectively. Therefore, detection of pulmonary nodules is considered a high-contrast task. It is shown in literature that DL algorithms generally maintain intermediate- and high-contrast spatial resolution performances compared with FBP and ASIRV 50\% at all doses levels ${ }^{24,26}$. Greffier et al reported higher 50\% task-based transfer function values (TTF $50 \%$ ) with DL than with IR from 15 to $2.5 \mathrm{mGy}$ and the opposite from 1 to $0.5 \mathrm{mGy}^{25}$. Our research shows no significant difference in pulmonary nodule detection between DL and IR reconstruction techniques. 
This study has some limitations. (1) Our research was conducted on a phantom with simulated nodules. These nodules are too uniform and spherical. Furthermore, our phantom study included nodules with different densities simulating solid and ground glass morphology, but it did not include subsolid nodules with part-solid morphology. Part of the phantom is composed by pulmonary vasculature and bronchial branching to simulate normal lung architecture, but the low density of the surrounding air is still different from that of normal lung tissue. (2) We focused on the impact of DLIR on visual detection of pulmonary nodules by radiologists. Regardless of the impact of dose, this task is prone to errors related to search, recognition and decision. We aimed to overcome this by including four independent readers. It is without doubt that Al-algorithms will play an important role in lung cancer screening, including computer-aided detection. The impact of DLIR on computer-aided detection needs to be unraveled. (3) This study investigated only the first step in the diagnostic algorithm of pulmonary nodules in chest CT, being detection of lung nodules. The impact of DLIR on nodule characterization, volumetric assessment and nodule morphology still needs to be investigated.

\section{CONCLUSIONS}

In conclusion, current study shows that TrueFidelity is non-inferior to ASIR-V for the clinical task of nodule detection in chest $\mathrm{CT}$, even at very low dose levels. 


\section{REFERENCES}

1. Hansell DM, Bankier AA, MacMahon H, McLoud TC, Müller NL, Remy J. Fleischner Society: glossary of terms for thoracic imaging. Radiology. 2008;246(3):697-722. doi:10.1148/radiol.2462070712

2. Bray F, Ferlay J, Soerjomataram I, Siegel RL, Torre LA, Jemal A. Global cancer statistics 2018: GLOBOCAN estimates of incidence and mortality worldwide for 36 cancers in 185 countries. CA Cancer J Clin. 2018;68(6):394-424. doi:10.3322/caac.21492

3. Larici AR, Farchione A, Franchi $P$, et al. Lung nodules: size still matters. European Respiratory Review. 2017;26(146):170025. doi:10.1183/16000617.0025-2017

4. Snoeckx A, Reyntiens $P$, Desbuquoit $D$, et al. Evaluation of the solitary pulmonary nodule: size matters, but do not ignore the power of morphology. Insights into Imaging. 2018;9(1):73 - 86. doi:10.1007/s13244-017-0581-2

5. Aberle DR, Adams AM, Berg CD, et al. Reduced lung-cancer mortality with low-dose computed tomographic screening. N Engl J Med. 2011;365(5):395-409. doi:10.1056/NEJMoa1102873

6. Koning $\mathrm{HJ}$ de, Aalst $\mathrm{CM}$ van der, Jong PA de, et al. Reduced Lung-Cancer Mortality with Volume CT Screening in a Randomized Trial. New England Journal of Medicine. 2020;382(6):503-513. doi:10.1056/nejmoa1911793

7. Pastorino U, Silva M, Sestini S, et al. Prolonged lung cancer screening reduced 10 -year mortality in the MILD trial: new confirmation of lung cancer screening efficacy. Ann Oncol. 2019;30(7):1162-1169. doi:10.1093/annonc/mdz117

8. Becker N, Motsch E, Trotter A, et al. Lung cancer mortality reduction by LDCT screening-Results from the randomized German LUSI trial. International Journal of Cancer. 2020;146(6):1503-1513. doi:10.1002/ijc.32486

9. Kauczor H-U, Baird A-M, Blum TG, et al. ESR/ERS statement paper on lung cancer screening. European Radiology. Published online 2020:1 - 18. doi:10.1007/s00330-020-06727-7

10. Kauczor H-U, Baird A-M, Blum TG, et al. ESR/ERS statement paper on lung cancer screening. European Respiratory Journal. 2020;55(2):1900506. doi:10.1183/13993003.00506-2019

11. Brenner DJ. Radiation risks potentially associated with low-dose CT screening of adult smokers for lung cancer. Radiology. 2004;231(2):440-445. doi:10.1148/radiol.2312030880

12. Prezzi $D$, Owczarczyk K, Bassett $P$, et al. Adaptive statistical iterative reconstruction (ASIR) affects CT radiomics quantification in primary colorectal cancer. Eur Radiol. 2019;29(10):5227-5235. doi:10.1007/s00330-019-06073-3

13. Solomon J, Mileto A, Nelson RC, Roy Choudhury K, Samei E. Quantitative Features of Liver Lesions, Lung Nodules, and Renal Stones at Multi-Detector Row CT Examinations: Dependency on Radiation Dose and Reconstruction Algorithm. Radiology. 2015;279(1):185-194. doi:10.1148/radiol.2015150892

14. Geyer LL, Schoepf UJ, Meinel FG, et al. State of the Art: Iterative CT Reconstruction Techniques. Radiology. 2015;276(2):339-357. doi:10.1148/radiol.2015132766

15. GE Healthcare. A new era of image reconstruction: TrueFidelity ${ }^{\mathrm{TM}}$ Technical white paper on deep learning image reconstruction. Available from: https://www.gehealthcare.com//jssmedia/040dd213fa89463287155151fdb01922.pdf. Published online July 1, 2019. Accessed $\begin{array}{llll}\text { October } & 1, & 2020 . & \text { https://www.gehealthcare.com/- }\end{array}$ /jssmedia/040dd213fa89463287155151fdb01922.pdf

16. Chakraborty DP, Berbaum KS. Observer studies involving detection and localization: modeling, analysis, and validation. Med Phys. 2004;31(8):2313-2330. doi:10.1118/1.1769352

17. Samei E, Bakalyar D, Boedeker KL, et al. Performance evaluation of computed tomography systems: Summary of AAPM Task Group 233. Medical Physics. 2019;46(11):e735-e756. doi:10.1002/mp.13763 
18. den Harder AM, Willemink MJ, de Ruiter $\mathrm{QMB}$, et al. Achievable dose reduction using iterative reconstruction for chest computed tomography: A systematic review. European Journal of Radiology. 2015;84(11):2307-2313. doi:10.1016/j.ejrad.2015.07.011

19. Crop A, Smeets $P$, Hoof T, et al. Correlation of clinical and physical-technical image quality in chest CT: A human cadaver study applied on iterative reconstruction. BMC medical imaging. 2015;15:32. doi:10.1186/s12880-015-0075-y

20. Miéville FA, Gudinchet F, Brunelle F, Bochud FO, Verdun FR. Iterative reconstruction methods in two different MDCT scanners: physical metrics and 4-alternative forced-choice detectability experiments-a phantom approach. Phys Med. 2013;29(1):99-110. doi:10.1016/j.ejmp.2011.12.004

21. De Marco P, Origgi D. New adaptive statistical iterative reconstruction ASiR-V: Assessment of noise performance in comparison to ASiR. J Appl Clin Med Phys. 2018;19(2):275-286. doi:10.1002/acm2.12253

22. Afadzi M, Lysvik EK, Andersen HK, Martinsen ACT. Ultra-low dose chest computed tomography: Effect of iterative reconstruction levels on image quality. Eur J Radiol. 2019;114:62-68. doi:10.1016/j.ejrad.2019.02.021

23. Euler A, Solomon J, Marin D, Nelson RC, Samei E. A Third-Generation Adaptive Statistical Iterative Reconstruction Technique: Phantom Study of Image Noise, Spatial Resolution, Lesion Detectability, and Dose Reduction Potential. American Journal of Roentgenology. 2018;210(6):1301-1308. doi:10.2214/AJR.17.19102

24. Solomon J, Lyu P, Marin D, Samei E. Noise and spatial resolution properties of a commercially available deep-learning based CT reconstruction algorithm. Med Phys. Published online June 7, 2020. doi:10.1002/mp.14319

25. Greffier J, Hamard A, Pereira F, et al. Image quality and dose reduction opportunity of deep learning image reconstruction algorithm for CT: a phantom study. Eur Radiol. Published online February 25, 2020. doi:10.1007/s00330-020-06724-w

26. Racine $D$, Becce $F$, Viry $A$, et al. Task-based characterization of a deep learning image reconstruction and comparison with filtered back-projection and a partial model-based iterative reconstruction in abdominal CT: A phantom study. Physica Medica: European Journal of Medical Physics. 2020;76:2837. doi:10.1016/j.ejmp.2020.06.004

27. Jensen CT, Liu X, Tamm EP, et al. Image Quality Assessment of Abdominal CT by Use of New Deep Learning Image Reconstruction: Initial Experience. Am J Roentgenol. 2020;215(1):50-57. doi:10.2214/AJR.19.22332

28. Kim I, Kang H, Yoon HJ, Chung BM, Shin N-Y. Deep learning-based image reconstruction for brain CT: improved image quality compared with adaptive statistical iterative reconstruction-Veo (ASIR-V). Neuroradiology. doi:10.1007/s00234-020-02574-x 
FIGURE LEGENDS

Figure 1. (left) internal structure of Kyoto Kagaku Lungman phantom showing the pulmonary vessels and (right) a selection of 9 round, solid nodules used to mimic pulmonary lesions.
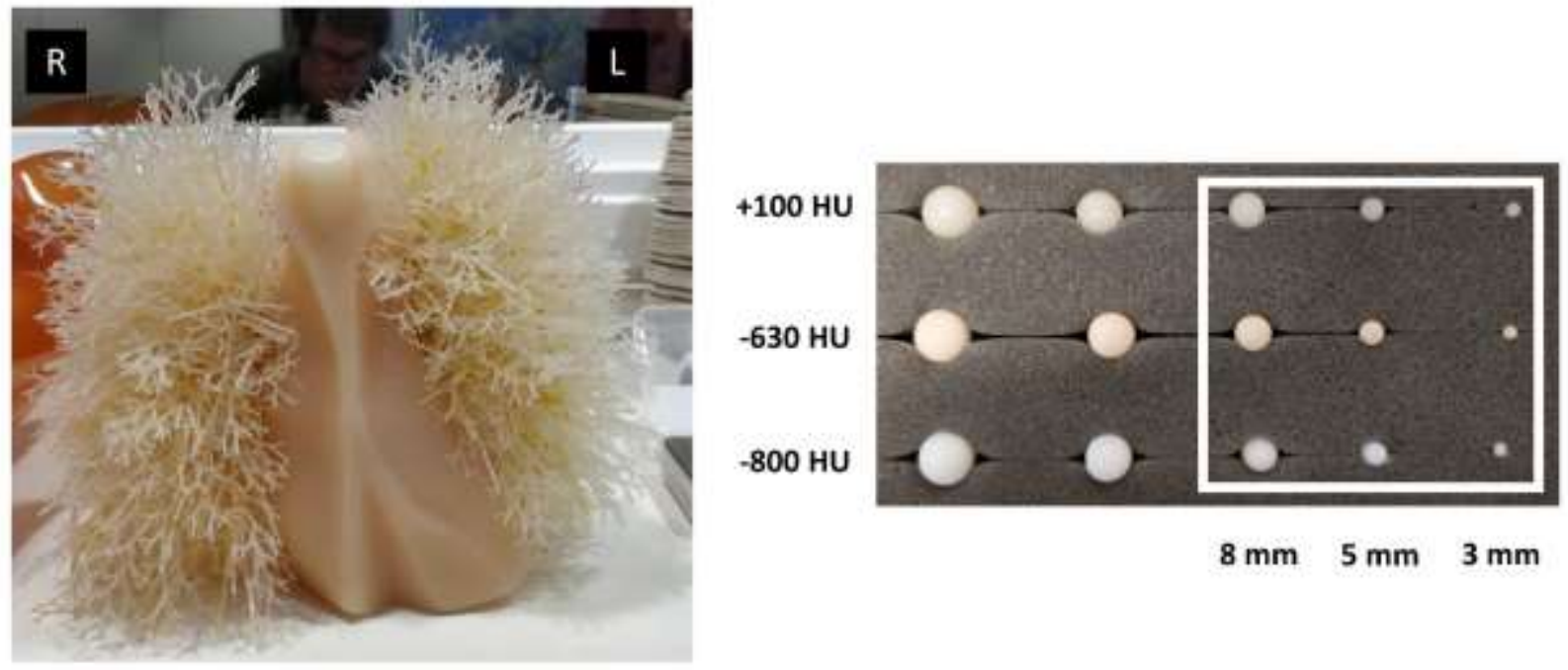
Table 1. Different configurations (16) of nodule placement in Lungman phantom.

\begin{tabular}{|c|c|c|c|c|c|c|c|}
\hline Configuration & Number of lesions & & & & ons* & & \\
\hline$A$ & 4 & $\mathrm{M} 8 \mathrm{~mm}$ & $\mathrm{H} 8 \mathrm{~mm}$ & $\mathrm{H} 5 \mathrm{~mm}$ & $\mathrm{M} \mathrm{5mm}$ & & \\
\hline B & 5 & $\mathrm{~L} 3 \mathrm{~mm}$ & $\mathrm{H} 8 \mathrm{~mm}$ & $\mathrm{M} 3 \mathrm{~mm}$ & $\mathrm{H} 3 \mathrm{~mm}$ & $\mathrm{M} 8 \mathrm{~mm}$ & \\
\hline C & 3 & $\mathrm{~L} 5 \mathrm{~mm}$ & H $8 \mathrm{~mm}$ & $\mathrm{H} 3 \mathrm{~mm}$ & & & \\
\hline $\mathrm{D}$ & 3 & $\mathrm{H} 5 \mathrm{~mm}$ & $\mathrm{~L} 8 \mathrm{~mm}$ & $\mathrm{~L} 5 \mathrm{~mm}$ & & & \\
\hline$E$ & 6 & L $8 \mathrm{~mm}$ & $\mathrm{~L} 5 \mathrm{~mm}$ & $\mathrm{~L} 3 \mathrm{~mm}$ & $\mathrm{H} 3 \mathrm{~mm}$ & $\mathrm{H} 5 \mathrm{~mm}$ & M $8 \mathrm{~mm}$ \\
\hline $\mathrm{F}$ & 3 & $\mathrm{M} 3 \mathrm{~mm}$ & $\mathrm{~L} 5 \mathrm{~mm}$ & $\mathrm{H} 5 \mathrm{~mm}$ & & & \\
\hline G & 4 & $\mathrm{M} 3 \mathrm{~mm}$ & $\mathrm{~L} 5 \mathrm{~mm}$ & $\mathrm{H} 8 \mathrm{~mm}$ & $\mathrm{~L} 3 \mathrm{~mm}$ & & \\
\hline $\mathrm{H}$ & 6 & $\mathrm{M} 5 \mathrm{~mm}$ & $\mathrm{~L} 8 \mathrm{~mm}$ & $\mathrm{H} 3 \mathrm{~mm}$ & $\mathrm{~L} 5 \mathrm{~mm}$ & $\mathrm{~L} 3 \mathrm{~mm}$ & $\mathrm{H} 5 \mathrm{~mm}$ \\
\hline I & 5 & $\mathrm{H} 3 \mathrm{~mm}$ & $\mathrm{~L} 8 \mathrm{~mm}$ & $\mathrm{M} 3 \mathrm{~mm}$ & $\mathrm{M} 8 \mathrm{~mm}$ & $\mathrm{H} 8 \mathrm{~mm}$ & \\
\hline J & 3 & $\mathrm{M} 8 \mathrm{~mm}$ & $\mathrm{~L} 5 \mathrm{~mm}$ & $\mathrm{M} 5 \mathrm{~mm}$ & & & \\
\hline$K-P$ & 0 & & & & & & \\
\hline
\end{tabular}

*L (low density, -800 HU); M (medium density, -630 HU); H (high density, +100 HU) 
Table 2. F-statistics (numerator, denominator, degrees of freedom) and p-values calculated for JAFROC statistical analysis.

\begin{tabular}{lcc}
\hline Statistical Analysis & F-statistics & p-value \\
& (degrees of freedom, numerator/denominator) & \\
\hline JAFROC & $0.05(3 / 61.9)$ & 0.987 \\
\hline
\end{tabular}


Table 3. JAFROC figures of merit (FoMs) for the detection of lung nodules on chest images processed with each of the investigated image reconstruction algorithms. The FoMs are reported for individual readers and for the average across readers.

\begin{tabular}{ccccc}
\hline & \multicolumn{4}{c}{ Reconstruction algorithm } \\
\cline { 2 - 5 } Observer & ASIR-V 50\% & DL-L & DL-M & DL-H \\
\hline 1 & 0.557 & 0.560 & 0.560 & 0.558 \\
2 & 0.548 & 0.558 & 0.557 & 0.557 \\
3 & 0.568 & 0.576 & 0.562 & 0.570 \\
4 & 0.550 & 0.553 & 0.552 & 0.547 \\
Average & 0.556 & 0.561 & 0.558 & 0.558 \\
\hline
\end{tabular}


Table 4. Difference in figure of merit between all modality pairings with $95 \%$ confidence intervals.

\begin{tabular}{ccc}
\hline Reconstruction algorithms & $\begin{array}{c}\text { Difference } \\
\text { in mean FoM }\end{array}$ & $\begin{array}{c}95 \% \mathrm{Cl} \text { of the difference } \\
\text { in FoM }\end{array}$ \\
\hline ASIR-V vs DL-L & -0.00535 & {$[-0.03417,0.02348]$} \\
ASIR-V vs DL-M & -0.00205 & {$[-0.03088,0.02678]$} \\
ASIR-V vs DL-H & -0.00219 & {$[-0.03088,0.02678]$} \\
DL-L vs DL-M & 0.00330 & {$[-0.02553,0.03213]$} \\
DL-L vs DL-H & 0.00315 & {$[-0.02567,0.03198]$} \\
DL-M vs DL-H & -0.00015 & {$[-0.02897,0.02868]$} \\
\hline
\end{tabular}

